\title{
COMMENTARY
}

\section{Hyperoxia after cardiac arrest may not increase ischemia-reperfusion injury}

\author{
Cornelia W Hoedemaekers and Johannes G van der Hoeven* \\ See related research by Bellomo et al., http://ccforum.com/content/15/2/R90
}

\begin{abstract}
In the last decade, moderate hypothermia has become the mainstay of treatment in the post-resuscitation period. However, for the damaged brain, optimizing oxygen transport, including arterial oxygenation, may also be important. The current view states that hyperoxia in the immediate post-resuscitation period may worsen cerebral outcome, and international guidelines recommend a target arterial oxygen saturation of $94 \%$ to $98 \%$. An article in the previous issue of Critical Care challenges this viewpoint. In an elegant study using a Cox proportional hazards model combined with sensitivity analyses and time period matching, the authors show no independent association between hyperoxia and in-hospital mortality. The present commentary discusses these contradictory findings and suggests a practical solution to solve these differences.
\end{abstract}

An article by Bellomo and colleagues [1] in the previous issue of Critical Care challenges the current view that hyperoxia in the immediate post-resuscitation period increases cerebral damage in cardiac arrest victims. These findings are at least remarkable and apparently contradict recent post-resuscitation guidelines.

Patients who initially survive an out-of-hospital cardiac arrest and are admitted to the ICU have a dismal prognosis. Despite the recent introduction of moderate therapeutic hypothermia, mortality is still approximately $65 \%$ to $70 \%$ [2]. The beneficial effects of moderate hypothermia are partly explained by the mitigating effects on ischemia-reperfusion injury. Consequently, other measures that may attenuate ischemia-reperfusion injury have

*Correspondence: hahoe@mac.com

Department of Intensive Care, Radboud University Nijmegen Medical Centre, P.O. Box 9101, 6500 HB, Nijmegen, The Netherlands been suggested. This includes the prevention of postresuscitation hyperoxia as the current evidence suggests that hyperoxia may worsen cerebral injury [3]. Animal experiments apparently confirm the detrimental effects of post-resuscitation hyperoxia on outcome. Hyperoxic reperfusion increases hippocampal neuronal death and induces behavioral deficits in a rat global cerebral ischemia model [4]. In a canine ventricular fibrillation cardiac arrest model, hyperoxic reperfusion decreased the activity of the hippocampal pyruvate dehydrogenase complex, possibly due to an increase in peroxynitrite levels [5] and impaired oxidative energy metabolism [6]. Finally, a clinically applicable protocol designed to reduce post-resuscitative hyperoxia in dogs by using oximetry and aiming for an oxygen saturation as measured by pulse oximetry $\left(\mathrm{SpO}_{2}\right)$ of $95 \%$ significantly reduced the number of injured $\mathrm{CA} 1$ neurons and improved the neurological deficit score [7]. The British Thoracic Society recently recommended that the inspired oxygen concentration be targeted to an arterial oxygen saturation of $94 \%$ to $98 \%$ [8]. This is also advocated in the most recent guidelines from the European Resuscitation Council [9].

Why then did Bellomo and colleagues arrive at a different conclusion? In an elegant retrospective analysis, they included 12,108 patients resuscitated from nontraumatic cardiac arrest. Hyperoxia was defined as an arterial partial pressure of oxygen $\left(\mathrm{PaO}_{2}\right)$ of greater than $300 \mathrm{~mm} \mathrm{Hg}$ in the first 24 hours after admission. Isolated hypoxemia was defined as a $\mathrm{PaO}_{2}$ of less than $60 \mathrm{~mm} \mathrm{Hg}$. A total of 1,285 (10.6\%) patients had hyperoxia and 1,168 (9.7\%) had isolated hypoxemia. Mortality was higher in the hyperoxia group compared with the normoxia group (59\% versus $47 \%$ ) but not compared with hypoxemia patients. Patients with isolated hypoxemia had the lowest discharge home rate (19\%). In a multivariate model, hyperoxia still had an odds ratio for hospital death of 1.2 ( $95 \%$ confidence interval of 1.1 to 1.6), but a Cox proportional hazards model combined with sensitivity analyses and time period matching showed no independent association between hyperoxia and in-hospital mortality. In 
contrast, in most of the models, isolated hypoxemia remained an independent risk factor for hospital mortality.

So where does this information leave us? Clearly, postresuscitation care is more than moderate hypothermia alone. Uncertainty still exists over the optimal blood pressure, the amount of cerebral blood flow, the hemoglobin level, the blood glucose regulation, and the partial pressure of arterial carbon dioxide $\left(\mathrm{PaCO}_{2}\right)$ and now also over the $\mathrm{PaO}_{2}$. Although the evidence up to now clearly suggested a detrimental effect of post-resuscitation hyperoxia, the weight of the present study raises serious doubts. Clearly, fear of hyperoxia should not lead to an indiscriminate decrease in inspired oxygen level, as hypoxemia is certainly bad for the damaged brain. On the other hand, hyperoxia had no proven beneficial effects either. Therefore, there is no reason to revise the current guidelines targeting a post-resuscitation arterial oxygen saturation of $94 \%$ to $98 \%$.

\section{Abbreviations}

$I \mathrm{CU}$, intensive care unit; $\mathrm{PaO}_{2}$, arterial partial pressure of oxygen.

\section{Competing interests}

The authors declare that they have no competing interests.

Published: 22 June 2011

\section{References}

1. Bellomo R, Bailey M, Eastwood GM, Nichol A, Pilcher D, Hart GK, Reade MC, Egi M, Cooper DJ, the Study of Oxygen in Critical Care (SOCC) Group: Arterial hyperoxia and in-hospital mortality after resuscitation from cardiac arrest. Crit Care 2011, 15:R90.

2. van der Wal G, Brinkman S, Bisschops LL, Hoedemaekers CW, van der Hoeven JG, de Lange DW, de Keizer NF, Pickkers P: Influence of mild therapeutic hypothermia after cardiac arrest on hospital mortality. Crit Care Med 2011, 39:84-88.

3. Kilgannon JH, Jones AE, Shapiro NI, Angelos MG, Milcarek B, Hunter K, Parrillo JE, Trzeciak S; Emergency Medicine Shock Research Network (EMShockNet) Investigators: Association between arterial hyperoxia following resuscitation from cardiac arrest and in-hospital mortality. JAMA 2010, 303:2165-2171.

4. Hazelton JL, Balan I, Elmer GI, Kristian T, Rosenthal RE, Krause G, Sanderson TH, Fiskum G: Hyperoxic reperfusion after global cerebral ischemia promotes inflammation and long-term hippocampal neuronal death. J Neurotrauma 2010, 27:753-762.

5. Richards EM, Rosenthal RE, Kristian T, Fiskum G: Postischemic hyperoxia reduces hippocampal pyruvate dehydrogenase activity. Free Radic Biol Med 2006, 40:1960-1970.

6. Richards EM, Fiskum G, Rosenthal RE, Hopkins I, McKenna MC: Hyperoxic reperfusion after global ischemia decreases hippocampal energy metabolism. Stroke 2007, 38:1578-1584.

7. Balan IS, Fiskum G, Hazelton J, Cotto-Cumba C, Rosenthal RE: Oximetryguided reoxygenation improves neurological outcome after experimental cardiac arrest. Stroke 2006, 37:3008-3013.

8. O'Driscoll BR, Howard LS, Davison AG; British Thoracic Society: BTS guideline for emergency oxygen use in adult patients. Thorax 2008, 63 Suppl 6:vi1-68.

9. Deakin CD, Nolan JP, Soar J, Sunde K, Koster RW, Smith GB, Perkins GD: European Resuscitation Council Guidelines for Resuscitation 2010 Section 4. Adult advanced life support. Resuscitation 2010, 81:1305-1352.

doi:10.1186/cc10250

Cite this article as: Hoedemaekers CW, van der Hoeven JG: Hyperoxia after cardiac arrest may not increase ischemia-reperfusion injury. Critical Care 2011, 15:166. 